\title{
EVALUACIÓN DE LA ADHESIÓN DE UN PEGANTE REALIZADO CON ALMIDÓN NATIVO DE YUCA (MANIHOT SCULENTA CRANTZ) VARIEDAD M-TAI
}

\author{
Evaluation of the accession of A native starch GLUE WORKED \\ WITH CASSAVA (MANIHOT SCULENTA CRANTZ) VARIETY M-TAI
}

Andrés Vergara N."

Moisés Hernández R."

Roberto Ramírez-Díaz

Recibido: 8 de noviembre de 2017

Aceptado: 23 de abril de 2018

\section{Resumen}

En este estudio se evaluó las fuerzas de adhesividad y de cáscara de un pegante. El pegante se realizó con almidón nativo de yuca (Manihot sculenta Crantz) variedad M-Tai y se usó hidróxido de sodio como agente hidrolizante. En la elaboración del pegante se evaluaron cinco soluciones de almidón nativo de yuca $(10,20,30,40$ y $50 \%$ p/v) y cuatro concentraciones de $\mathrm{NaOH}(0,0.66$, 1.33 y $2 \%$ p/p). Los resultados indicaron que las uniones papel-papel, cartón-cartón y papelvidrio, realizadas con los pegantes hechos con $40 \%-2 \%, 40 \%-0 \%$ y $50 \%-0 \%$ p/v-p/p de almidón de yuca y $\mathrm{NaOH}$, respectivamente, presentaron mayor fuerza de adhesión. Las uniones papelpapel, cartón-cartón y papel-vidrio que mostraron mayor fuerza de fricción, como un indicativo de mayor durabilidad del pegamento, fueron los pegantes realizados con $50 \%-2 \%$, $50 \%-1.33 \%$ y $50 \%-2 \%$ p/v-p/p de almidón y $\mathrm{NaOH}$, respectivamente. El pegante a base de almidón de yuca no presentó diferencias significativas $(\mathrm{p}<0.05)$ con tres adhesivos comerciales (Colbocol, Supertack y Pegajoso), teniendo mejor adhesividad y durabilidad en las uniones hechas con los sustratos papel-papel y papel-vidrio.

Palabras clave: pegante, agente hidrolizante, adhesividad, cáscara, sustratos.

\footnotetext{
* Máster en dirección de operaciones y logística, Barcelona, España. Vicerrectoría de CTel, Corporación Universitaria del Caribe CECAR, Sucre, Colombia. https://orcid.org/0000-0003-3391-9235. andres.vergaran@ cecar.edu.co ${ }^{* \star}$ Máster en Ciencias Ambientales. Vicerrectoría de CTel, investigador Junior, Grupo De Investigación Salud Pública, Corporación Universitaria del Caribe-CECAR, Sucre, Colombia. https://orcid.org/0000-0001-9285-3438.

Moises.hernadez@cecar.edu.co

*** Ing. agroindustrial. Grupo de investigación Desarrollo e Innovación de Procesos Alimentarios (DESINPA), Facultad de Ingeniera, Universidad de Sucre, Sucre, Colombia. https://orcid.org/0000-0002-3937-235X. robertocradiz19@live.com
} 


\section{Abstract}

In this study, the adhesive and shell forces of a glue were evaluated. The glue was made with native cassava starch (Manihot sculenta Crantz) variety M-Tai and sodium hydroxide was used as a hydrolyzing agent. In the preparation of the glue, five solutions of native cassava starch were evaluated $(10,20,30,40$ and $50 \% \mathrm{w} / \mathrm{v})$ and four concentrations of $\mathrm{NaOH}(0,0.66,1.33$ and $2 \% \mathrm{w} / \mathrm{w})$. The results indicated that the paper-paper, cardboard-paperboard and paper-glass joints, made with the adhesives made with $40 \%-2 \%, 40 \%-0 \%$ and $50 \%-0 \% \mathrm{w} / \mathrm{v}-\mathrm{w} / \mathrm{w}$, of cassava starch and $\mathrm{NaOH}$ respectively, showed higher adhesion strength and the paper-paper, cardboard-paperboard and paper-glass joints, which showed greater frictional force as an indication of greater durability of the glue, were the glued with $50 \%-2 \%, 50 \%-1.33 \%$ and $50 \%-2 \%$ w/v - w/w, of starch and $\mathrm{NaOH}$ respectively. The paste based on cassava starch did not present significant differences $(\mathrm{p}<0.05)$ with three commercial adhesives (Colbocol, Supertack and Pegajoso), having better adhesiveness and durability in the bonds made with paper-paper and glass-paper substrates

Keywords: adhesive, hydrolyzing agent, adhesive, shell, substrates.

\section{INTRODUCCIÓN}

Uno de los productos más importantes obtenidos a partir de la yuca es el almidón. El almidón es un polímero natural que presenta ventajas sobre algunos otros materiales empleados en la industria, ya que proviene de reservas naturales, es renovable y biodegradable (Sánchez, 2007; Luna y Mera, 2006). En el sector industrial, el almidón nativo de yuca se emplea principalmente en la fabricación de papel, la preparación de pegantes, la perforación de pozos petroleros y la fabricación de dinamita, así como en la industria textil y de alimentos preparados. Según Aristizábal y Sánchez (2007), las propiedades adhesivas del almidón y algunos almidones modificados "se usan para la elaboración de adhesivos para diversas aplicaciones en la industria de papel y cartón ya sea para adhesivos de alta fuerza adhesiva o para colas" (p. 47), principalmente por su bajo costo y naturaleza hidrofílica.

Estudios realizados han revelado que los adhesivos y pegantes hechos a partir de raíces y tubérculos son más fluidos. Además, se ha demostrado que estas sustancias tienen menor tendencia a la retrogradación (Aristizábal, 2004). Los almidones con alto contenido en amilopectina permiten obtener adhesivos mucho más elásticos, brillantes y transparentes.

Los almidones más usados son los obtenidos a partir de la yuca y la papa. Sin embargo, el almidón de yuca tiene algunas ventajas sobre el almidón de papa que se confieren a los adhesivos, como mayor viscosidad y estabilidad, mayor maleabilidad para trabajar, olor y sabor neutros y posibilidad de ser combinados fácilmente con emulsiones sintéticas. Otra característica importante que presentan los pegantes a base de almidón de yuca es que las uniones que se realizan con el pegado presentan mayor resistencia a la tracción que las uniones realizadas con el adhesivo derivado del almidón de papa (Aristizábal, 2004).

Dentro de los parámetros de calidad de los pegantes, se encuentra que, después de pasado el proceso de endurecimiento, las uniones resultantes deben tener "ciertas características químicas y mecánicas para ser confiable. Entre estas, una de las más importantes es la fuerza adhesiva, es decir, la fuerza con que se mantienen unidas las dos superficies" (Arias y Claro, 2006, p. 37).

Otro parámetro importante de calidad es la durabilidad del pegamento. La prueba de cáscara produce la información usada para la determinación de este parámetro. En la prueba de cáscara, 
la fuerza se aplica en un ángulo que simula el retiro del material adhesivo. La prueba puede determinar la fuerza de fricción que ejerce el adhesivo al pegarse al material (Garde, 2006). La adición de agentes químicos al almidón puede ayudar en las propiedades del pegante, como es el caso del hidróxido de sodio $(\mathrm{NaOH})$, cuya función consiste en disminuir y regular la temperatura de gelatinización del almidón, lo que permite un pegado más rápido y aumenta la viscosidad y la capacidad de penetración del pegante (Chávez, 2012). El objetivo de este trabajo es evaluar las fuerzas de adhesividad y de cáscara de un pegante realizado con almidón nativo de yuca (Manihot sculenta Crantz) variedad M-Tai utilizando hidróxido de sodio como agente hidrolizante.

\section{Metodología}

\section{Materiales}

El almidón empleado en el experimento fue almidón nativo, obtenido en la planta piloto de procesos agroindustriales de la Universidad de Sucre, Colombia, a partir de yuca variedad M-Tai. Se siguió la metodología descrita por Alarcón y Dufour (1998) para la obtención del almidón de la yuca.

\section{Elaboración del pegante}

La elaboración del pegante se realizó a partir de diferentes soluciones de almidón, a las que se les adicionó $\mathrm{NaOH}$ como agente hidrolizante en diferentes concentraciones. Se utilizaron cinco soluciones de almidón nativo de yuca $(10,20,30,40$ y $50 \%$ p/v) y cuatro concentraciones de $\mathrm{NaOH}$ $(0,0.66,1.33$ y $2 \% \mathrm{p} / \mathrm{p})$. Las combinaciones resultantes fueron puestas en reactores de un litro por un tiempo de 16 minutos y a temperatura controlada para mantener a $5 \pm 2{ }^{\circ} \mathrm{C}$ por debajo de la temperatura de gelatinización del almidón de yuca (gelatinización a los $66{ }^{\circ} \mathrm{C}$ ) (Aristizábal y Sánchez, 2007). Los reactores estaban equipados con agitación mecánica.

\section{Pruebas de calidad del pegante}

Los pegantes fueron probados en los siguientes sustratos: papel bond blanco, cartón corrugado y lámina de vidrio transparente liso. Las uniones realizadas fueron papel-papel, cartón-cartón, papelvidrio. El tiempo esperado después de aplicar los pegantes en forma de película delgada en las uniones a temperatura ambiente fue de 5, 10 y 15 minutos, respectivamente, que corresponde al tiempo de secado de los pegantes en las tres uniones. Posteriormente, se realizaron las siguientes pruebas:

\section{Pruebas de adhesividad o extensible (ángulo 90\%)}

Se ejerció una fuerza perpendicular a las uniones a las que se les aplicó el pegamento. Se siguió el procedimiento descrito por Garde (2006) y la norma UNE-EN 12960 de 2001 (Asociación Española de Normalización y Certificación, 2001). El pegamento se aplicó en forma de película sobre el sustrato colocado de manera fija sobre un bloque de madera fijado al piso; otro bloque de menos peso con la otra parte del sustrato fue colocado encima del bloque inferior, haciendo la respectiva unión de los sustratos. Los bloques ejercieron una presión uniforme en el área de unión. Luego del tiempo de secado respectivo para cada tipo de unión, los bloques fueron separados por un sistema de poleas y motor que se unió al bloque de madera superior. Un dinamómetro situado entre la primera polea y el bloque de madera se utilizaron para medir la carga máxima ejercida en la separación de los bloques (expresada en Newton, N). El tiempo que tardó en despegarse se calculó usando un cronómetro; este tiempo se midió desde cuando las cuerdas estaban tensas, hasta cuando se separaron los bloques y, por ende, la unión de los sustratos pegados. 


\section{Prueba de cáscara (ángulo 45)}

Se realizó el mismo procedimiento descrito anteriormente, pero el bloque inferior fue ajustado para variar el ángulo de despegue hasta que la cuerda y los bloques unidos formaran un ángulo de $45^{\circ}$.

Durante los ensayos, el pegamento se aplicó a la superficie del bloque inferior, teniendo en cuenta que el adhesivo estaba uniformemente distribuido.

\section{Comparación de los pegantes con adhesivos comerciales}

Se compararon los pegantes que tuvieron mejores resultados en la prueba de adhesividad y de cáscara con tres adhesivos comerciales de uso común. Se les aplicó las mismas pruebas con los sustratos utilizados en este estudio, realizando las uniones de acuerdo a la metodología ya descrita.

Para realizar el análisis estadístico, se planteó un diseño completamente al azar con arreglo factorial de dos factores: solución de almidón en cinco niveles (10,20, 30 ,40 y $50 \%$ p/v) y concentración de $\mathrm{NaOH}$ en cuatro niveles $(0,0.66,1.33$ y $2 \% \mathrm{p} / \mathrm{p})$, con tres repeticiones por tratamiento. Se aplicó un análisis de varianza (ANOVA) para determinar diferencias significativas entre los tratamientos y la prueba de Tukey con un nivel de significancia del $5 \%$. Para el análisis de los datos se usó el programa estadístico InfoStat.

\section{Resultados}

\section{Prueba de adhesividad o extensible}

En la figura 1 se muestran los resultados de la fuerza de adhesión (expresada en N) que ejerce el pegante para los tres tipos de unión que se realizaron. El análisis de varianza (ANOVA) evidencia que las soluciones de almidón y las concentraciones de $\mathrm{NaOH}$ presentaron un efecto significativo $(\mathrm{p}<0.05)$ para los tres tipos de unión (papel-papel, cartón-cartón y papel-vidrio).



Figura 1. Fuerza adhesiva para las uniones con relación a soluciones de almidón y concentraciones de $\mathrm{NaOH}$.

En la figura 1 se observa que las uniones papel-papel, cartón-cartón y papel-vidrio, realizadas con los pegantes hechos con $40 \%-2 \%, 40$ \%-0 \% y 50 \%-0 \% p/v-p/p de almidón y $\mathrm{NaOH}$, 
respectivamente, presentaron mayor fuerza de unión entre los sustratos. Este comportamiento puede atribuirse a que los demás pegantes después de ser secados lograron un anclaje mecánico que fue mucho más débil, posiblemente por una excesiva penetración del mismo en los sustratos, lo que provocó que la película en la superficie del sustrato fuera deficiente, es decir, que las fuerzas intermoleculares entre los dos materiales fueran menores (Arias y Claro, 2006). La unión cartóncartón presentó mayor fuerza de adhesión, lo que puede deberse a que existe mayor afinidad del adhesivo con el adherente. Según Arias y Claro (2006; ver López, 2005) en las uniones, además de las fuerzas mecánicas, se presentan

Diferentes fuerzas de atracción de las moléculas del adhesivo hacia el adherente, que dan paso a la formación de fuerzas secundarias o de Van der Waals, entre las moléculas de los dos materiales, que hace posible la unión de acuerdo con la naturaleza [y afinidad] del adherente y del adhesivo. (pp. 34-35)

En la figura 1 se observa que (de acuerdo al tipo de unión) las bajas concentraciones de $\mathrm{NaOH}$ generan una mayor fuerza de adhesividad entre los dos sustratos; esto puede deberse a que las mayores concentraciones de $\mathrm{NaOH}$ provocan en el pegante mayor viscosidad, lo que daría lugar a que no penetre fácilmente y no se distribuya de forma regular sobre la superficie (López, 2005). Las soluciones de almidón entre el $40 \%$ y $50 \%$ p/v fueron las que tuvieron mayor fuerza de atracción para mantener unidos los sustratos, lo que es consecuencia de las fuerzas adhesivas; igualmente, según el análisis estadístico, estas soluciones presentaron las medias más altas.

\section{Prueba de cáscara}

En la figura 2 se muestran los resultados de la fuerza de fricción que ejerce el adhesivo al pegarse al sustrato, los cuales fueron determinados a través de la prueba de cáscara.

\section{Cáscara}

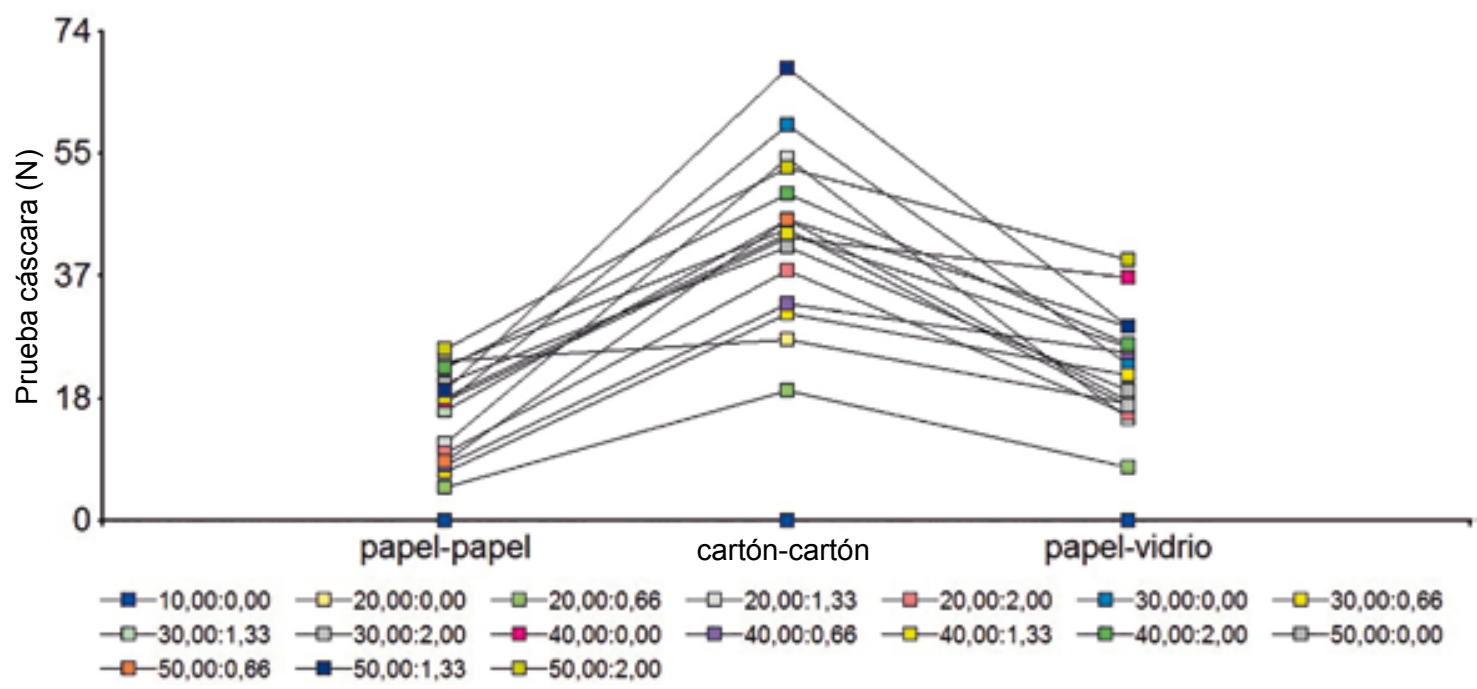

Figura 2. Prueba de cáscara para las uniones con relación a soluciones de almidón y concentraciones de $\mathrm{NaOH}$. 
$\mathrm{El}$ análisis de varianza evidencia que las soluciones de almidón tuvieron un efecto significativo $(\mathrm{p}<0.05)$ para los tres tipos de unión (papel-papel, cartón-cartón y papel-vidrio) y que el $\mathrm{NaOH}$ no ejerció un efecto significativo $(\mathrm{p}<0.05)$ en la unión papel-vidrio. En la figura 2 se observa que las uniones papel-papel, cartón-cartón y papel-vidrio, realizadas con el pegante hecho con $50 \%-2 \%, 50 \%-1.33 \%$ y $50 \%-2 \%$ p/v-p/p de almidón y $\mathrm{NaOH}$, respectivamente, fueron las que presentaron mayor fuerza de unión entre los sustratos; igualmente, la mayor fuerza de fricción al aplicarle una fuerza externa para romper la unión formada entre ellos indica mayor durabilidad del pegamento. Estos pegamentos presentaron las medias más altas en relación a los otros pegamentos formulados de acuerdo a la prueba de Tukey. Las uniones hechas con $10 \% \mathrm{p} / \mathrm{v}$ de almidón y $2 \% \mathrm{p} / \mathrm{p}$ de $\mathrm{NaOH}$ en los sustratos presentaron menores fuerzas, lo que puede ocurrir porque tienen menor calidad y es más fácil retirarlos el pegamento (Garde, 2006).

\section{Comparación de los pegantes con adhesivos comerciales}

En la tabla 1 se mostraron los pegantes de almidón de yuca que tuvieron mayor fuerza en la prueba de adhesividad y de cáscara y de tres adhesivos comerciales comunes, usados a nivel doméstico y en la industria maderera.

$\mathrm{El}$ análisis de varianza realizado a los pegamentos y los tres adhesivos comerciales no presentó diferencias significativas $(\mathrm{p}<0.05)$ en las pruebas de adhesividad y cáscara aplicadas en los tres diferentes sustratos. Estos resultados indican que el pegamento realizado a base de almidón de yuca podría llegar a ser un buen sustituto de estos adhesivos comerciales en los sustratos empleados en este estudio. El pegante realizado también mostró medias más altas que los tres adhesivos comerciales.

En la tabla 1 se observa que el pegamento a base de almidón de yuca, de acuerdo a las pruebas realizadas, tuvo en términos generales mejores características de adhesividad y de durabilidad en las uniones hechas con los sustratos papel-papel y papel-vidrio, lo que puede deberse a que este pegamento presenta mayor afinidad con la estructura de estos sustratos que los adhesivos comerciales, por lo que genera mayor fuerza de adhesión y resistencia a la tracción

Tabla 1. Prueba de adhesividad y de cáscara para pegantes de almidón de yuca y de adhesivos comerciales

\begin{tabular}{lllccc}
\hline \multirow{2}{*}{ Adhesivo } & \multicolumn{5}{c}{ Prueba de adhesividad y cáscara } \\
\cline { 2 - 5 } & \multicolumn{1}{c}{ Sustrato } & Ángulo & Fuerza (N) & Angulo & Fuerza (N) \\
\hline \multirow{2}{*}{$\begin{array}{l}\text { Pegante de } \\
\text { almidón de yuca }\end{array}$} & Papel-papel & $90^{\circ}$ & $31.33 \pm 3.06$ & $45^{\circ}$ & $26.00 \pm 2.00$ \\
& Cartón-cartón & $90^{\circ}$ & $78.33 \pm 2.89$ & $45^{\circ}$ & $68.33 \pm 2.89$ \\
& Papel-vidrio & $90^{\circ}$ & $49.00 \pm 3.61$ & $45^{\circ}$ & $39.33 \pm 2.31$ \\
Colbocol & Papel-papel & $90^{\circ}$ & $18.67 \pm 1.15$ & $45^{\circ}$ & $10.00 \pm 0.00$ \\
& Cartón-cartón & $90^{\circ}$ & $55.33 \pm 5.03$ & $45^{\circ}$ & $40.00 \pm 2.00$ \\
& Papel-vidrio & $90^{\circ}$ & $40.67 \pm 9.02$ & $45^{\circ}$ & $16.33 \pm 3.21$ \\
Supertack & Papel-papel & $90^{\circ}$ & $26.67 \pm 5.77$ & $45^{\circ}$ & $11.67 \pm 2.89$ \\
& Cartón-cartón & $90^{\circ}$ & $90.00 \pm 0.00$ & $45^{\circ}$ & $76.67 \pm 5.77$ \\
& Papel-vidrio & $90^{\circ}$ & $20.33 \pm 4.04$ & $45^{\circ}$ & $37.33 \pm 0.58$ \\
Pegajoso & Papel-papel & $90^{\circ}$ & $38.33 \pm 2.89$ & $45^{\circ}$ & $13.33 \pm 2.89$ \\
& Cartón-cartón & $90^{\circ}$ & $80.00 \pm 0.00$ & $45^{\circ}$ & $50.00 \pm 0.00$ \\
& Papel-vidrio & $90^{\circ}$ & $36.67 \pm 2.89$ & $45^{\circ}$ & $20.67 \pm 1.15$ \\
\hline
\end{tabular}




\section{Conclusiones}

Las uniones papel-papel, cartón-cartón y papel-vidrio, realizadas con los pegamentos hechos con $40 \%-2 \%, 40 \%-0 \%$ y $50 \%-0 \%$ p/v-p/p de almidón de yuca y $\mathrm{NaOH}$, respectivamente, tuvieron mayor fuerza de adhesión, lo que pudo ser causado por una mayor afinidad del adhesivo con el adherente. Mientras que las uniones papel-papel, cartón-cartón y papel-vidrio, que ejercieron mayor fuerza de fricción al aplicarle una fuerza externa para romper la unión formada, fueron los pegamentos realizados con $50 \%-2 \%, 50 \%-1.33 \%$ y $50 \%-2 \%$ p/v-p/p de almidón y $\mathrm{NaOH}$, respectivamente. El pegamento a base de almidón de yuca presentó mejores características de adhesividad y de durabilidad en las uniones hechas con los sustratos papel-papel y papel-vidrio.

\section{REFERENCIAS}

Alarcón, F. y Dufour, D. (1998). Almidón agrio de yuca en Colombia: producción y recomendaciones. Tomo I: Producción y recomendaciones. Cali, Colombia: CIAT y CIRAD.

Arias, D., y Claro, J. (2006). Evaluación de cinco conservantes adicionados sobre diferentes tipos de almidones para ser empleados en procesos de restauración en el patrimonio documental (tesis de pregrado). Microbiología Industrial, Facultad de Ciencias, Pontificia Universidad Javeriana, Bogotá, Colombia.

Aristizábal, G. (2004). Estudio de la viabilidad técnica y económica de la producción de dextrina a partir de yuca utilizando tecnologías de vía seca. Palmira, Colombia: CIAT.

Aristizábal, J. y Sánchez, T. (2007). Guía técnica para producción y análisis de almidón de yuca (Boletín de Servicios Agrícolas de la FAO n. ${ }^{\circ}$ 163). Roma: FAO.

Asociación Española de Normalización y Certificación. (2001). UNE-EN 12960: adhesivos para papel y cartón, embalaje y productos sanitarios desechables: determinación de la resistencia a la cizalla. Madrid: Aenor

Chávez, D. (2012). Elaboración de goma modificada a partir de la dextrina de yuca para uso en la industria cartonera (tesis de pregrado). Facultad de Ingeniería Química, Universidad de Guayaquil, Guayaquil, Ecuador.

Garde J. (2006). Colas y adhesivos. Recuperado de http://www.guiaenvase.com/bases/guiaenvase. nsf/V02wn/Colas\%20y\%20adhesivos?OpenDocument

López, C. (2005). Evaluación del almidón de yuca dulce como posible adhesivo para procesos de intervención de obras con soporte en papel (trabajo de grado). Facultad de Bienes Muebles, Universidad Externado de Colombia, Bogotá, Colombia.

Luna, W., y Mera, J. (2006). Producción de dextrinas de yuca a partir de almidón nativo en la rallanderia Todoyuca ubicada en el corregimiento Pescador (municipio de Caldono, departamento del Cauca, Colombia) (tesis de pregrado). Facultad de Ciencias Agropecuarias, Universidad del Cauca, Popayán, Colombia.

Sánchez, I. (2007). Propiedades fisicoquimicas de almidones catiónicos elaborados por extrusión (tesis de maestría). Instituto Politécnico Nacional, México D. F., México. 
